\title{
Heinrich Küper : le Blanc dont on parle à mi-voix (Gupuna, Santa Ana, sud-est des îles Salomon)
}

\section{Sandra Revolon}

\section{(2) OpenEdition \\ 12 Journals}

Édition électronique

URL : http://journals.openedition.org/jso/1199

DOI : $10.4000 /$ jso. 1199

ISSN : $1760-7256$

Éditeur

Société des océanistes

\section{Édition imprimée}

Date de publication : 1 juin 2003

Pagination : 65-75

ISSN : 0300-953x

\section{Référence électronique}

Sandra Revolon, « Heinrich Küper : le Blanc dont on parle à mi-voix (Gupuna, Santa Ana, sud-est des îles Salomon) », Journal de la Société des Océanistes [En ligne], 116| Année 2003-1, mis en ligne le 26 mai 2008, consulté le 22 avril 2019. URL : http://journals.openedition.org/jso/1199 ; DOI : 10.4000/ jso. 1199

(c) Tous droits réservés 


\title{
Heinrich Küper : le Blanc dont on parle à mi-voix (Gupuna, Santa Ana, sud-est des îles Salomon)
}

par

\author{
Sandra REVOLON *
}

\section{RÉSUMÉ}

Entre le milieu des années 1910 et 1950, l'année où il mourut, Heinrich Küper, déserteur et aventurier allemand, vécut à Gupuna, un village de l'île de Santa Ana, au sud-est de l'archipel des îles Salomon. Conjuguant une attitude autoritaire, parfois violente, à un sens $d u$ commerce et à une connaissance fine de la culture et des représentations locales du pouvoir, il devint au fil des années un leader respecté par l'ensemble des habitants. Principalement ethnographique, cet article retrace l'histoire peu ordinaire de cet ancien militaire et expose les circonstances dans lesquelles il accéda au statut d'arafa, de " grand » au sein d'une communauté mélanésienne. Il s'interroge aussi sur l'héritage laissé par Küper qui, plus de trente années durant, fut le vecteur d'idées et de pratiques nouvelles, sur la configuration sociale et symbolique de ce village.

Mots-CLÉS: îles Salomon, histoire, changement social.

Peuplée de mille six cents horticulteurs et pêcheurs mélanésiens parlant le owa, une langue austronésienne, la petite île corallienne de Santa Ana est située dans la province de Makira, au sud-est des îles Salomon. Entre les deux guerres et jusqu'en 1950, elle abrita un hôte peu commun en la personne de Heinrich Küper, un déserteur et aventurier allemand originaire de Hambourg qui prit peu à peu sur la population locale un ascendant qu'il conserva trois décennies durant.

\section{ABSTRACT}

Between the mid 1910's and 1950, the year of his death, Heinrich Küper, German deserter and adventurer, lived in Gupuna, a village on the island of Santa Ana, in the south east of the Solomon Islands. Combining an authoritarian, sometimes violent, style with a trade spirit and a highly refined knowledge of local culture and representations of power, he became over the years a leader well respected by the inhabitants.

Mainly ethnographical, this article traces the uncommon history of this former military and reveals how he acceded to the status of arafa and became a figure of authority within a Melanesian community. The article also discusses the heritage left by Küper who, during more than thirty years, generated and inspired ideas and new practices influencing the social and symbolic configuration of this village.

KeYwORDS : Solomon Islands, history, social change.

Entre mythe et réalité, l'évocation de son souvenir s'accompagne aujourd'hui encore d'un certain malaise, sans doute par égard pour ses nombreux descendants, mais aussi parce que, un demi-siècle après sa mort, son autorité semble encore indirectement s'exercer sur les habitants du village de Gupuna où il résida. La durée de son séjour ainsi que la nature et l'intensité de son implication dans la vie locale font de la rencontre fortuite des gens de Santa Ana avec l'Allemand

* Centre de Recherche et de Documentation sur l'Océanie (CREDo), Campus Saint Charles, 3 Place Victor Hugo, 13331Marseille Cedex3 
un cas bien particulier de découverte rapide, et en partie initiée par un Européen, de l'État, de l'Église et du marché par un peuple mélanésien, Küper étant à la fois le vecteur d'idées et de pratiques nouvelles et un acteur éminent de leur mise en œuvre.

Le premier volet de cet article est consacré au récit des circonstances dans lesquelles un ancien militaire allemand devint le leader incontesté d'un village mélanésien. La question de l'héritage laissé par Küper sera ensuite abordée, où l'on tentera d'analyser l'influence encore décelable aujourd'hui de cet aefaka - cet homme blanc - sur la configuration sociale et culturelle de ce village ${ }^{1}$.

\section{L'installation de Küper à Gupuna}

En 1893 fut proclamé le British Solomon Islands Protectorate ${ }^{2}$, officiellement pour mettre fin aux activités des blackbirders, ces recruteurs de main-d'œuvre forcée pour les plantations de coton et de canne à sucre de Fidji et du Queensland qui menaçaient de réduire considérablement une population déjà atteinte par un état de guerre endémique et par les épidémies introduites par les Occidentaux.

Sous l'influence de l'administration coloniale et des grandes compagnies, l'exploitation de coprah se développa considérablement dès la fin du XIX ${ }^{\mathrm{e}}$ siècle aux îles Salomon, notamment dans les régions de Malaita, de Guadalcanal, des îles Shortland et de Makira, la grande île immédiatement au nord de Santa Ana ( $c f$. cartes 1 et 2).

Réunissant toutes les conditions qu'affectionnaient les marchands, Santa Ana fut elle aussi le théâtre de cette nouvelle activité. Sa population réduite limitait les risques de conflit avec les Européens et facilitait les négociations. La baie protégée de Gupuna, son village principal, permettait un bon ancrage et constituait un abri sûr en cas de gros temps ( $c f$. carte 3 ).

Sa situation géographique centrale facilitait de surcroît la collecte et le transport des marchandises dans toute la région, si bien qu'avec Ugi, située au nord-ouest de Santa Ana, la petite île devint le centre des activités commerciales dans le sud-est de l'archipel (Bennett, 1987 : 63).

À partir de 1882 et au cours des décennies qui suivirent, des Européens attirés par les perspectives économiques qu'offrait l'exploitation des cocoteraies commencèrent à s'installer à Santa Ana, mais l'alcool, l'isolement ou des affections liées au climat eurent raison de leur ténacité, si bien que leur séjour ne se prolongea pas au-delà de quelques années ${ }^{3}$. En 1919, un rapport administratif recensait dix-neuf Européens dans le district de Makira, parmi lesquels douze Anglais, quatre Français (deux pères et deux sœurs de la mission catholique romaine) et deux Allemands, ainsi qu'un Américain, tous marchands ou missionnaires, à l'exception du district officer de l'administration coloniale ${ }^{4}$.

L'un des deux Allemands identifiés s'appelait Heinrich Küper ${ }^{5}$. Cet officier de la Marine Impériale semble avoir déserté peu avant 1910 alors qu'il naviguait le long des côtes du sud-est de l'archipel (Golden, 1993 : 300). Il s'installa d'abord à Cape Surville, à l'extrême-sud de Makira, où il créa une cocoteraie d'une trentaine d'hectares. Son entreprise devint vite profitable, mais la Première Guerre mondiale éclata et, sur ordre des Anglais, il dut quitter sa plantation pour rejoindre Tulagi ${ }^{6}$, où furent rassemblés les quelques Allemands qui se trouvaient aux îles Salomon en cette période de troubles. Après quelques mois passés dans un camp surnommé le « Potsdam » et resté célèbre dans la région pour son ambiance festive et ses beuveries

1. Cet article a été rédigé à partir de données recueillies lors d'enquêtes de terrain réalisées en pidgin salomonais entre 1996 et 2002 sur les îles de Santa Ana (6 mois entre 1996 et 1997) et Aorigi (Santa Catalina) (16 mois entre 1998 et 2002) ainsi qu'à Honiara, la capitale des îles Salomon, où j'ai séjourné entre 2000 et 2002. A ces données ethnographiques s'ajoutent des informations tirées des archives coloniales britanniques conservées aux Archives Nationales à Honiara et des écrits d'auteurs qui se sont intéressés à cette région, notamment Bernatzik (1935a, 1935b, 1935c, 1936, 1952), Davenport (1972, 1980), Fox (1967), Golden (1993) et Mead (1973a, 1973b, 1973c). Ces recherches ont pu être menées grâce au soutien financier du Ministère des Affaires Étrangères et de la Fondation Lavoisier.

2. Le 6 octobre 1893, le Gouvernement britannique proclama le British Solomon Islands Protectorate sur Guadalcanal, Ngella, Savo, Makira et la Nouvelle-Géorgie. En 1898 vinrent s'ajouter les îles de Santa Cruz et Reef Islands, Taumako, Utupua, Vanikoro, Tikopia, Anuta et Rennell et Bellona, puis, en 1900, Isabel, Luaniua (Ontong Java), les îles Shortland et Choiseul, qui étaient jusqu'alors sous le contrôle de l'Allemagne. Bougainville et Buka furent rattachées à l'Australie.

3. Les frères Mac Donald vécurent à Gupuna entre 1882 à 1885, Charles Sproul et William Heughan entre 1882 et 1883 , Franck Nybert entre 1884 et 1892, Charles Olsen entre 1894 et 1897. Comme F. Nybert, il mourut à Gupuna, ainsi que Tom Butler, qui vécut sur l'île entre 1899 et 1918 (Golden, 1993).

4. British Solomon Islands Protectorate File 9/III — Eastern District Annual Reports 1919-1956 : Annual Report 1919.

5. Sa présence à Gupuna est mentionnée par Bernatzik (1935a, 1935b), Golden (1993) et Van den Broek (1939), qui rencontra Küper lors du voyage autour du monde qu'il effectua avec quatre autres Français à bord de la Korrigane entre 1934 et 1936. Les archives coloniales britanniques conservées à Honiara font également régulièrement cas de lui dès 1919.

6. Tulagi (Central province) fut la capitale des îles Salomon jusqu'en mai 1942, date à laquelle elle fut détruite par les bombardements des Japonais qui voulaient y établir une base d'hydravions. 

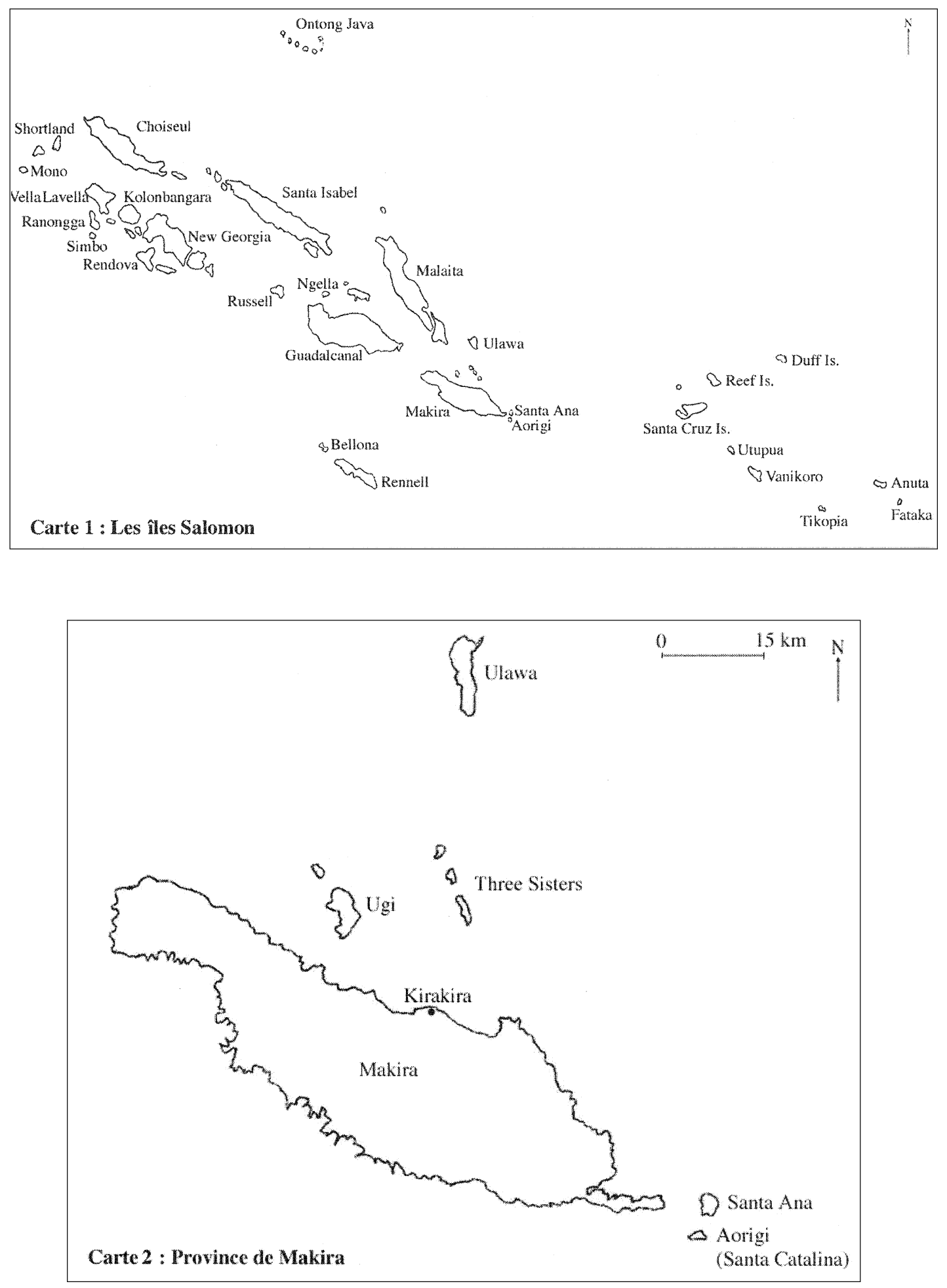

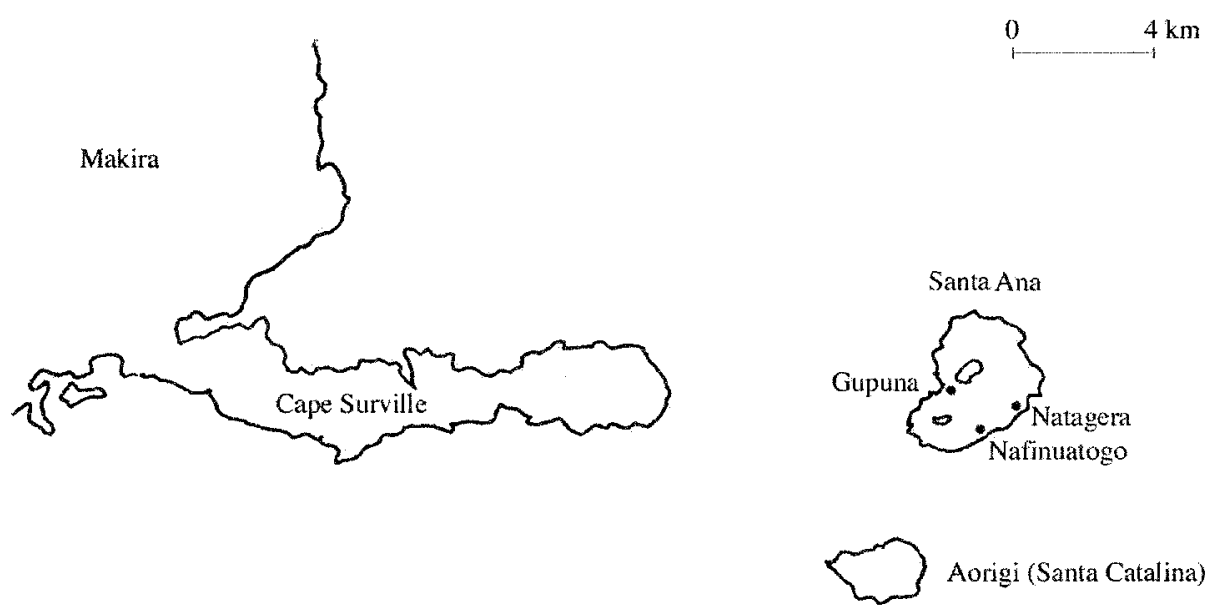

Carte 3 : Sud-est de la province de Makira

mémorables, Küper fut finalement relâché sur la promesse qu'il ne collaborerait pas avec l'ennemi et put rejoindre Makira (Golden, 1993 : 300). Un peu plus tard, pourvu de matériel de campement et d'une arme, il vint s'établir sur une pointe isolée de Santa Ana. Informés de la sévérité des expéditions punitives britanniques à l'encontre des communautés qui s'étaient montrées hostiles envers les Blancs, les habitants tolérèrent sa présence ${ }^{7}$.

Quelques mois après son installation, Küper voulut épouser Kafagamurironga, fille d'un homme éminent du clan Gafe, l'un des plus puissants de l'île ${ }^{8}$. Devant les hommes menaçant de l'attaquer s'il ne s'acquittait pas du prix de la fiancée, il sortit une arme et n'hésita pas à tirer pour les mettre en fuite. Quelques jours plus tard, il paya au père de la jeune femme une compensation matrimoniale substantielle composé d'objets manufacturés (Bernatzik, 1935a : 8). Le couple s'établit alors dans le village de Gupuna ${ }^{9}$. Un premier fils naquit en 1917 , suivi plus tard de deux autres garçons puis d'une fille. À l'époque de la naissance de son premier enfant, Küper avait déjà une certaine influence au sein du clan de sa femme. Il permit notamment à un missionnaire de la South Sea Evange- lical Mission originaire de Makira de s'installer à Gupuna en lui cédant une terre du clan Gafe, geste qu'il regretta lorsqu'il vit s'édifier une église là où il croyait voir construire une école pour ses enfants. S'érigeant alors en partisan des traditions locales, il tenta de persuader les habitants de rejeter l'étranger, mais ses efforts furent vains et il assista, impuissant, aux débuts de l'évangélisation du village ${ }^{10}$.

\section{Une ascension progressive}

Les années passant, Küper développa un intérêt certain pour les coutumes locales, au point qu'il publia un article ethnographique (Küper, 1926). En 1921, il parvint à convaincre les habitants de Nafinuatogo, un village situé de l'autre côté de l'île, de s'élever temporairement contre l'installation de missionnaires anglicans ${ }^{11}$. Sa connaissance des mœurs de ses hôtes fut aussi pour lui la condition nécessaire à la réalisation de ses plans personnels, singulièrement lorsqu'il entreprit d'exercer son autorité au-delà des limites du clan Gafe et de l'étendre à l'ensemble des habitants de Gupuna, dans des circonstances que l'on va à présent s'attacher à décrire. Il fut

7. Sur ces expéditions punitives, $c f$. notamment Corris et Keesing 1980.

8. Ainsi que l'indiquait Mead (1973c : 215) : « The clans of Santa Ana [...] are ranked, the highest social and ritual position being enjoyed by Gafe and Garofai clans. These two clans regard themselves not only as the founding clans of Santa Ana [...] but also as the descendants of chiefs »".

9. Pour autant que l'on puisse en juger sur les photographies des demeures de chacun (Golden, $1993: 300$; Gash et Witthaker, 1975 : 41), la maison de Küper n'avait aucun rapport avec la somptueuse résidence de la célèbre Queen Emma à Ralum (Nouvelle-Bretagne).

10. British Solomon Islands Protectorate File 9/III — Districts Reports 1919-1965 : Eastern District Monthly and Quaterly reports : Monthly Report August 1920 et Gapiara (1999).

11. Ils finirent par s'y établir en 1925 (Fox, 1967). 
bientôt considéré comme le chief priest du village (Bernatzik, 1935a : 8) et se composa un statut hybride qui semblait s'inspirer tout à la fois du mwane apuna, expert en religion et en médecine, du mwane ni mae, chef de guerre dont on craignait la violence, du mwane ni farunga enfin, en se faisant organisateur d'échanges et de festins. Sa supériorité reposait sur les qualités requises par les Owa pour devenir un homme important, mais alors que l'autorité d'un tel arafa (littéralement «grand ») s'exerçait dans des domaines habituellement plutôt séparés et mobilisant des aptitudes différentes, l'aefaka, lui, semblait les rassembler toutes.

Küper devint d'abord l'homme le plus riche de l'île. Il était l'époux d'une femme fortunée et influente qui disposait des nombreuses terres de son clan, de monnaies de perles de coquillages et de cochons en grande quantité, mais, propriétaire d'une grande plantation de cocotiers à Makira, il possédait également sur cette grande île et à Santa Ana plusieurs comptoirs où il vendait ou troquait du tabac et des objets manufacturés contre des trocas, de l'écaille de tortue, des bêches-de-mer et des coquillages précieux. En 1926, il acheta l'unique cocoteraie de Santa Ana aux frères Mac Donald, des Canadiens qui avaient eux-mêmes exploité la plantation entre 1882 et 1885 avant de se retirer (Golden, 1993 : 299). Il reprit également à son seul profit le commerce des danses, en organisant des spectacles sur les navires de guerre qui commencèrent à venir visiter Gupuna dès le milieu du second conflit mondial. En 1940, au retour de sa tournée d'inspection, le fonctionnaire britannique en poste à Kirakira, la capitale de la province de Makira, évoquait la grogne des danseurs qui se plaignaient de ne pas être payés en retour des prestations arrangées par Küper ${ }^{12}$.

À cette puissance économique s'ajoutèrent le respect et la crainte qu'inspira la brutalité dont l'Européen fit preuve pour gérer ses affaires et régler les conflits. On dit aujourd'hui qu'il n'hésita pas à frapper et à menacer de ses armes des hommes d'Aorigi (Santa Catalina), l'île voisine ( $c f$. carte 3 ), venus lui vendre une tortue, pour la simple raison que leur insistance l'indisposait. On évoque aussi les insultes qu'il lui était fréquent de proférer à l'encontre de ceux qui travaillaient pour lui lorsqu'ils peinaient à maîtriser les instruments mis à leur disposition. Une anecdote fameuse raconte ainsi comment des hommes employés au séchage du coprah confon- dirent l'aiguille d'une balance posée près d'eux avec celle de l'horloge, censée rythmer leur temps de travail et de pause : abusés par l'immobilité de ladite aiguille, ils travaillèrent sans relâche, tout le jour. Le soir venu, ils durent encore encaisser les sarcasmes de leur employeur, excédé par leur ignorance.

Küper eut également recours à la violence pour s'assurer le monopole du commerce local de biens européens et mettre un terme aux échanges entre les habitants et les marchands qui passaient dans la région. Au début des années 1930, il annonça même que quiconque serait surpris en train de négocier avec ceux-ci serait exécuté sur le champ (Bernatzik, 1935a: 8) Des anciens de Gupuna m'ont dit que peu après cette menace, Küper tua un marchand chinois lors d'une altercation. Ces événements ne figurent dans aucun rapport d'archives mais on considère aujourd'hui sur l'île que son initiative mit fin au passage des commerçants itinérants.

Jusqu'à la pacification des îles Salomon par les Anglais dans les années 1930, la violence constituait chez les Owa un moyen d'acquérir du prestige, à l'image des chefs de guerre (arafa ni mae, littéralement " homme de combat »), et même de s'enrichir, dans le cas des tueurs à gages (arafa ni watewate, littéralement " homme à gages ») qui recevaient un " prix de l'homme ${ }^{13}$ » en échange du meurtre qu'ils commettaient sur une victime désignée par un commanditaire. Sans s'impliquer, bien sûr, dans de telles activités, Küper fit preuve d'emportements qui sont encore célèbres dans la région et, s'ils font souvent rire aujourd'hui, il semble qu'ils n'aient pas à l'époque suscité d'opposition de la part des habitants. Les raisons de ce consentement apparent paraissent claires : l'aefaka était armé et bénéficiait de surcroît de la protection des administrateurs coloniaux.

Nouvelle manifestation de son prestige - ou de son autorité - ou rumeur infondée, dans sa chronique des pionniers européens aux îles Salomon, Golden rapporte que l'un des rôles que s'était attribué l'Allemand était de déflorer les jeunes vierges avant leur mariage :

« According to Tom Elkington junior, Küper was de facto king of Santa Ana and that role carried with it certain responsibilities, which included the responsibility of initiating young virgins into womanhood. Tom Elkington ${ }^{14}$ recalled that in July 1930 he was at

12. British Solomon Islands Protectorate File 9/III — Eastern District Annual Reports 1919-1956 : Resident Commissioner's Tour Sept/oct 1940.

13. Défini par Daniel de Coppet (de Coppet et Zemp, 1978 : 116) chez les 'Are'are de Malaita.

14. T. Elkington et E. Palmer dirigeaient à cette époque une compagnie de recrutement (Golden, 1993 : 301). 
Waimamurawith Küper, F.M. Campbell ${ }^{15}$ and others when they heard on the radio that Don Bradman scored 334 in the test at Leeds, Küper promptly announced that his score of virgins was in excess of 334. Ernie Palmer confirmed that he had often seen young girls been dragged by their fathers screaming to Küper's house for that very purpose » (Golden, 1993 : 299).

Interrogés sur ce sujet, les anciens de Santa Ana n'ont pas donné foi à ces allégations. Ils m'ont par ailleurs indiqué que, même s'il est culturellement admis que les jeunes filles aient des relations sexuelles avant le mariage, il n'existe pas - ni n'existait - de pratique de défloration rituelle des jeunes filles sur cette île. De plus, s'il a eu lieu, cet usage est difficilement imaginable à une telle échelle. En 1926, la population de Santa Ana s'élevait à 360 habitants et n'était que de 745 personnes en 1971 (Mead, 1973b : 82). Au-delà du cynisme de l'anecdote, le chiffre avancé par Elkington semble donc démesuré. Il reste que, tout autant que les récits des Owa, les témoignages laissés par les Occidentaux qui le connurent entretiennent le caractère pour le moins ambigu du personnage.

\section{Des relations privilégiées avec les esprits}

Si des aspects de sa personnalité conférèrent à sa domination un caractère despotique, Küper sut aussi se montrer sous un jour plus altruiste dans certaines occasions, ce qui contribua sans aucun doute à son ascension sociale. Il fut ainsi apprécié pour les connaissances médicales acquises lors de sa vie militaire, qu'il mit à profit en dispensant des soins aux malades qui sollicitaient son aide. En 1929, il interrompit ainsi une épidémie de coqueluche en distribuant des médicaments qu'il avait reçus de l'administration coloniale ${ }^{16}$. Peu à peu, les habitants lui accordèrent leur confiance et finirent même par refuser les soins du médecin détaché par les Britanniques qui faisait de temps à autre une tournée d'inspection à Santa Ana. L'accession à un statut comparable à celui des guérisseurs (mwane apuna ${ }^{17}$ ) conféra alors à l'Européen une autorité nouvelle qui grandit encore lorsque, le 4 octobre 1931, un violent tremblement de terre vint secouer la région. Après la première secousse, et vingt-quatre heures durant, la terre continua de trembler tous les quarts d'heure. Une lame de fond s'abattit sur les villages côtiers de la péninsule de Makira, où elle atteignit dix mètres de haut et se fit sentir jusqu'à cinq kilomètres à l'intérieur des terres, mais aussi à Santa Ana et à Aorigi, distante de trois kilomètres. Dix-huit villages furent détruits à Makira, et l'une des pointes d'Aorigi s'effondra dans l'océan ${ }^{18}$. Le niveau de Santa Ana baissa de trente centimètres selon le district officer, qui évalua le nombre de morts à une cinquantaine, pour une population totale de 1149 personnes dans le district ${ }^{19}$. Terrifiés, les habitants pensèrent être victimes de la colère des esprits et s'attendirent à voir leur île engloutie d'un instant à l'autre par les flots. Au milieu du chaos, Küper se fit rassurant et prédit un retour au calme (Bernatzik, 1935a : 9). Finalement, les secousses cessèrent, les eaux s'apaisèrent, l'orage qui accompagnait le tremblement de terre se dissipa et les habitants furent convaincus que tout cela était le résultat de l'action magique de l'Allemand (Bernatzik, 1935a : 9). Considéré comme capable de connaître l'avenir par divination et d'intervenir auprès des esprits des défunts pour obtenir leur aide, il consolida sa réputation de mwane apuna, d'expert en rituels.

Mais, pour les habitants de Gupuna, les relations que Küper entretenait avec le monde de l'invisible comportaient une autre dimension bien plus redoutable. Toujours à demi-mot, on lui attribue aujourd'hui des liens avec des ataro aera, des « esprits sauvages » et malfaisants, souvent évoqués lorsque sont commentés les comportements de certains membres du clan Gafe. On dit par exemple que Faupuru, un esprit sauvage, fut domestiqué par les descendants de l'aefaka. Portant bottes, pantalon, chemise et casquette de policier, il erre encore la nuit à la recherche d'une victime sur le chemin principal de l'île ou sur ses lieux favoris - la piste d'aviation ou l'école secondaire. Le rencontrer peut s'avérer fatal pour les vivants; on lui attribue aussi de nombreux viols. Gardien des biens des Gafe, il punit également de mort quiconque tente de faire main basse sur leurs richesses. Notons que si les agissements de Faupuru ne déparent pas des actions communément attribuées aux esprits

15. En 1917, F.M. Campbell était le premier district officer de Makira où il dirigea la construction de la station de l'administration coloniale à Kirakira. Il fut également propriétaire de diverses plantations entre 1920 et 1953, toujours dans la région de Makira (Golden, 1993 : 301).

16. British Solomon Islands Protectorate File 9/III - Eastern District Annual Reports 1919-1956 : Annual Report 1930.

17. Littéralement «homme interdit », mwane apuna désigne à la fois les guérisseurs et les spécialistes religieux que l'on considère comme des médiateurs entre les vivants et le monde des esprits.

18. Le niveau moyen d'Aorigi baissa de soixante-dix centimètres lors de ce tsunami (British Solomon Islands Protectorate File 9/III — Eastern District Annual Reports 1919-1956 : Annual Report 1932).

19. British Solomon Islands Protectorate File 9/III — Eastern District Annual Reports 1919-1956 : Annual Report 1932. 
sauvages, il s'en distingue cependant nettement de par son apparence, qui dénote sans doute l'influence de Küper sur les gens de Gupuna. Généralement décrits comme semblables aux hommes ou aux femmes de l'île, les esprits sauvages apparaissent en effet généralement vêtus d'un pagne, d'un short ou d'une jupe. Habillé à l'occidentale, coiffé de l'emblème d'une nouvelle forme d'autorité et familier de lieux chargés de sens par la modernité - la piste d'aviation et l'école - Faupuru rassemble quant à lui des éléments résultant de changements dont Küper fut l'instigateur à Gupuna.

Pour les Owa, le succès d'une entreprise dépend principalement du bon vouloir des esprits et de la qualité des relations qu'un homme entretient avec eux. Comparé aux Occidentaux qui l'avaient précédés et qui, dans des délais assez courts, quittèrent Santa Ana ou y moururent, faute de s'adapter à des conditions de vie inconfortables, la volonté inébranlable et les ambitions toujours accomplies de cet étranger-là durent offrir un contraste saisissant et faire forte impression sur les habitants. En conjuguant les succès d'ordre économique, médical et divinatoire à une grande robustesse physique, à un courage et à une force morale remarquables, Küper dut apparaître comme un véritable prodige dans l'art de communiquer avec les habitants de l'invisible et dans la manière de satisfaire leurs exigences pour s'assurer leur coopération à chacune de ses nouvelles initiatives. Du point de vue owa, l'intimité particulière liant l'Allemand et le clan Gafe avec le monde des esprits contribue aujourd'hui pour une large part à expliquer la réussite sociale de Küper. Elle éclaire aussi les raisons pour lesquelles son souvenir continue d'inspirer aujourd'hui de la crainte. En témoigne le sursaut d'une informatrice lorsqu'une luciole, considérée comme une manifestation de la présence de l'esprit sauvage Taririu, entra dans sa maison alors qu'elle évoquait le souvenir de l'Allemand. Interprétant ce signe comme un avertissement, elle changea promptement de sujet.

\section{« Celui dont le nom est célèbre » : Küper organi- sateur d'échanges}

Spécialiste religieux et guerrier à ses heures, il restait à Küper à endosser le rôle d'arafa $n i$ farunga, d'organisateur d'échanges et dispensa- teur de festins, pour réunir en lui les trois grandes figures d'autorité qui, jusqu'au $\mathrm{XIX}^{\mathrm{e}}$ siècle, se partagèrent le pouvoir politique dans cette région ${ }^{20}$. C'est ce qu'il fit en 1943 lorsqu'il décida d'organiser pour son fils aîné et pour les garçons de sa génération ce qui furent les dernières initiations masculines de Gupuna ${ }^{21}$. Mead (1973b) rapporte que les enfants furent reclus dans la maison cérémonielle de Natagera, le troisième village de l'île et que, six mois durant, Küper se chargea de coordonner les préparatifs de la fête de clôture. Une plate-forme entièrement peinte et sculptée de huit mètres de hauteur et de près de quatre-vingt mètres carrés de superficie fut construite pour l'occasion sur la place centrale de Gupuna. Très élaborée, elle témoignait autant du talent de ses concepteurs, notamment les sculpteurs qui furent payés pour participer à sa construction et à sa décoration, que de l'investissement financier de son instigateur. Des parents et amis des îles d'Ulawa, de Makira et d'Aorigi furent conviés à se joindre aux festivités, ainsi que des militaires de l'armée américaine alors stationnés à Guadalcanal. Au matin du grand jour, et avec le même anachronisme que l'irruption d'un hélicoptère au mariage de Peau d'Âne ${ }^{22}$, le général Howard et le commandant Wilson gagnèrent Santa Ana l'un en hydravion, l'autre dans un sous-marin de chasse. Montés à bord d'une pirogue de guerre, ils débarquèrent sur la plage de Gupuna où des guerriers les accueillirent par une mise en scène des combats d'autrefois. Les Américains furent ensuite rejoints par des fonctionnaires britanniques chargés de les escorter. L'épouse de Küper les conduisit sur la place du village où des habitants donnèrent une sorte de pièce de théâtre racontant l'asservissement des Salomonais par les Japonais puis leur libération par l'armée américaine. Des danseurs venus d'Ulawa furent accueillis à leur tour par l'épouse de Küper. Comme le veut la coutume, leurs prestations furent payées par leurs hôtes en monnaies de perles de coquillages et de dents de chauvesouris, de chiens et de dauphins.

L'après-midi, la procession des initiés s'ébranla et gravit les marches de la plate-forme, au sommet de laquelle les jeunes garçons, le corps couvert de parures précieuses, apparurent aux yeux de tous après six mois d'absence. Jusque tard dans la nuit, les danses des uns et des autres furent admirées et la nourriture partagée.

20. Sur la question du système politique à trois branches dans la région linguistique de Makira-Malaita, voir notamment Burt 1994, Hogbin 1939, Keesing 1985 et White 1991.

21. Mead (1973b) a consacré un article à la description détaillée de ces dernières initiations.

22. Dans le film de Jacques Demy (1971). 
Nul doute que la présence des soldats américains et des fonctionnaires anglais conféra à cette cérémonie un caractère particulièrement atypique, encore renforcé par la saynète relatant les méfaits des Japonais. Cependant, la présence et le nombre de ces aefaka que les insulaires considéraient comme leurs sauveurs furent considérés comme une preuve de l'étendue de l'influence de Küper et de sa famille et, à travers eux, de la renommée de toute la population de l'île qui les avait accueillis. De même, tout en affirmant la fortune des Küper, les dimensions considérables de cette fête, la richesse d'ornementation de la plate-forme, l'abondance des mets et la présence de danseurs accrurent aussi le prestige et la notoriété de Santa Ana. Les habitants de l'île ne manquèrent pas d'apprécier cette réussite, ce qui les incita peut-être à ne pas s'opposer aux innovations que Küper apporta au rituel qu'il était en train d'organiser. En fin connaisseur de la culture owa, et grâce à la mise sur pied de ces initiations, Küper s'attribua le rôle de tarofa katana (littéralement « le nom cité », expression qui désigne " celui dont le nom est célèbre »). Son nom fut en effet sur toutes les lèvres cette année-là et le prestige qu'il retira de l'événement fut considérable. Il accéda ainsi au statut de mwane arafa, d'" homme grand », dont la puissance économique fut reconnue de tous.

\section{Épilogue}

À la fin de sa vie, Küper s'opposa aux partisans du Maasina Ruru ${ }^{23}$. Ici encore, les versions sont multiples et se contredisent car, pour certains, il se contenta de prévenir les instigateurs du mouvement à Santa Ana des risques qu'ils encouraient en participant à de telles activités, mais pour d'autres, il fut celui qui informa de leur identité les autorités britanniques établies à Kirakira, provoquant leur incarcération. Il mourut brutalement en 1950, laissant derrière lui une île profondément différente de celle qu'il avait trouvée à son arrivée.

Sur le plan économique, le clan Gafe bénéficia particulièrement de son alliance avec l'Allemand, qui lui permit de renforcer sa supériorité sur les autres clans en présence ${ }^{24}$. À la mort de Küper, ses descendants devinrent propriétaires de cocoteraies de 6,5 hectares à Santa Ana et de
28 hectares à Cape Surville (Makira), ainsi que des comptoirs qu'il possédait sur les deux îles. Ils héritèrent aussi des contacts nombreux que Küper avait entretenus avec les autorités locales, les missions et les marchands, autrement dit avec les Européens de toute la région, et jusque dans la capitale. Leur évidente prospérité d'aujourd'hui montre que ces précieux atouts leur permirent de devancer sans effort les autres habitants sur le chemin de la modernité : ils dirigent notamment l'unique commerce d'exportation de langoustes vers la capitale et pourvoient aisément aux frais de scolarité de leurs enfants, dont certains poursuivent des études au sein d'universités australiennes et néo-zélandaises.

Son fils aîné, Geoffrey Küper, prit le relais des activités de son père (y compris son intérêt pour l'ethnologie, comme en témoigne l'article qu'il publia en 1937 sur les tatouages à Santa Ana). Il semble qu'il hérita aussi de son autorité, qu'il exerça en appliquant peu ou prou les mêmes méthodes que son défunt père, comme en témoigne cette dernière anecdote. Lassés d'entendre les villageois se plaindre des dégâts causés par les vaches que son père avait introduites quelques années auparavant, Kafagamurironga Küper et ses fils posèrent en 1957 un grillage embrassant la moitié des maisons de Gupuna et une ceinture arborée. Devant le mécontentement des habitants, Kafagamurironga se déclara propriétaire des terres de toute l'île et annonça que, par conséquent, aucune compensation ne serait payée pour dédommager les dégâts causés par ses bêtes dans les jardins plantés autour des habitations, pas plus que pour l'usage abusif qu'elle faisait des terres d'autrui. Son fils aîné ajouta que toutes les maisons situées à l'intérieur de l'enclos devaient être déplacées dans un délai de douze heures, au terme duquel elles seraient brûlées. Alerté par les villageois consternés, le district officer porta le cas devant un tribunal d'Honiara, la capitale, qui mit les Küper en demeure de retirer la clôture et de compenser les dégâts occasionnés par les bêtes ${ }^{25}$. Cependant, rien ne bougea et, en mars 1958, James Tedder, le district commissioner de Kirakira, semblait en proie à un profond découragement, si l'on en croit les termes par lesquels il concluait un courrier adressé au commissaire chargé de la gestion des terres: "Have you any suggestions or war-

23. Le mouvement de protestation à l'encontre du gouvernement britannique qui conduisit les îles Salomon à leur indépendance en 1978. Sur ce sujet, voir notamment Keesing 1978 et de Coppet et Zemp 1978.

24. Le mode de descendance est matrilinéaire à Santa Ana.

25. British Solomon Islands Protectorate File 9/II - General Correspondance 1956-62 : Santa Ana Land Disputes 1957-58 — Letter from the District Commissioner S.R. Pearson to Juridical Commissioner, October 1957. 
nings for this nice job ? » ${ }^{26}$. La Cour de Justice d'Honiara enjoignit les Küper à se plier au jugement et, finalement, la clôture fut démontée et les bêtes tuées.

Les écrits des anthropologues ayant travaillé sur cette île dans les années 1960-1970 évoquent à plusieurs reprises la présence de Geoffrey Küper à Gupuna (Davenport, 1972 ; Mead, 1973a et b). Il était apparemment difficile d'effectuer des recherches à Santa Ana mais aussi à Aorigi sans qu'il ne tente d'y participer - et d'y exercer un certain contrôle - en indiquant par exemple aux chercheurs l'endroit où s'installer, les gens avec qui il était préférable de travailler, ou encore en offrant ses services pour les traductions. Il finit par quitter Gupuna pour s'établir à Honiara où il mourut à son tour en 1989 (Golden, 1993 : 300). Ses deux frères et sa sœur avaient quant à eux quitté l'île après la mort de leur père.

\section{Conclusion : l'héritage de l'aefaka à Gupuna}

L'histoire de l'installation de Küper et de son intégration à la population de Gupuna semble indiquer que la violence employée comme unique moyen de persuasion n'aurait pas permis à l'aefaka d'acquérir l'importance politique qu'il avait à la fin de sa vie. C'est en la conjuguant avec d'autres attitudes, notamment une connaissance fine de la culture owa et la capacité à comprendre et à s'adapter aux représentations locales du pouvoir, qu'il parvint à s'imposer comme un leader à part entière. Fort de la position éminente d'un genre nouveau qu'il acquit au fil des années, et en dépit de ses déclarations en faveur de la pérennité des coutumes locales, en précipitant dans un temps très court (trois décennies seulement) la conversion au christianisme, l'accès à l'éducation et l'ouverture à une économie de marché, Küper a pu, en partie involontairement, contribuer à faire d'un ensemble d'idées et de pratiques nouvelles, sans doute jusque-là connues seulement par ouï-dire, un moteur de transformations de l'organisation sociale et symbolique locale.
Gupuna se distingue aujourd'hui des villages voisins par un désir de modernité plus apparent qu'ailleurs. Expression de cette volonté de changement : un tiers de la population de Kirakira est originaire de ce village et, en 1998, une centaine de familles de Gupuna vivaient à Honiara, contre seulement seize personnes originaires d'Aorigi ${ }^{27}$. L'argent envoyé aux parents par ceux qui ont fait le choix de partir travailler en milieu urbain et semi-urbain fait d'ailleurs de Gupuna l'un des villages les plus riches de Makira, comme en témoignent la forte concentration de maisons en matériaux de construction modernes que l'on y rencontre, les dizaines de bateaux à moteur qui s'alignent sur les plages de la baie, ou encore sa coopérative ${ }^{28}$ et ses commerces ${ }^{29}$, attestant du pouvoir d'achat des habitants.

Mais Gupuna se caractérise aussi par la perte de nombreux éléments de la culture traditionnelle, notamment des pratiques collectives majeures, comme les initiations masculines, les cérémonies de secondes funérailles ou les scarifications faciales, qui ne sont plus pratiquées depuis près d'un demi-siècle, alors même qu'elles continuent d'être réalisées par les habitants d'Aorigi et d'Ulawa (carte 2).

Enfin, interrogés sur la question de la relative insécurité qui règne aujourd'hui à Gupuna, mes interlocuteurs n'attribuèrent pas la perte de légitimité des dirigeants locaux à l'influence des Anglais qui colonisèrent les îles Salomon durant quatre-vingts ans, ou à celle de l'Église évangéliste (South Sea Evangelical Church), ouvertement opposée aux pratiques coutumières et à laquelle s'est convertie la majorité des habitants du village. Ce qui leur paraissait plus pertinent ici était la présence d'immigrants - jamais personnellement désignés - dont les descendants refusèrent, dit-on, de se soumettre à l'autorité des chefs, ce qui aurait entraîné une dégradation sensible et récente de l'ordre social. Chacun déplore les écarts qui ponctuent aujourd'hui la vie du village et lui confèrent une image désastreuse désormais bien établie dans toute la région ${ }^{30}$.

26. British Solomon Islands Protectorate File 9/II — Letter Tedder to Allen, Special Lands Commissioner, Honiara, March 1958.

27. Ces chiffres n'ont pas été actualisés depuis le coup d'État survenu dans la capitale en juin 2000. La population d'Aorigi s'élève à environ huit cents personnes.

28. À Gupuna, la coopérative est le principal magasin de l'île. Pour en faire partie, les familles versent une cotisation puis perçoivent en fin d'année un pourcentage des bénéfices, calculé à partir de leurs propres dépenses au cours de l'année. On y vend des denrées alimentaires (boîtes de viande et de poissons, riz, etc.) et des fournitures diverses (fil de pêche, hameçons, lampes à pétrole, tissu, tabac, parfois des timbres, etc.), de nature et quantité variables selon le ravitaillement, dont la fréquence est aléatoire.

29. En 1998, on en dénombrait huit pour les huit cents habitants que compte le village, où l'on vendait principalement des produits alimentaires (conserves de poisson, riz, soupes « chinoises », biscuits) et/ou du pain.

30. En décembre 2000, un jeune homme ivre blessa grièvement à coups de machettes son oncle maternel et l'un de ses cousins. En 1998, des garçons violèrent une jeune fille de quatorze ans, originaire de l'ouest de l'archipel et élève de l'école secondaire, où 
Sans être la cause unique de ces bouleversements, Küper a sans doute participé à transformer en profondeur l'ordre établi, les pratiques et le système de pensée des habitants de Gupuna pour en faire un village fortement acculturé où une plus grande qualité de la vie matérielle et économique semble aller de pair avec une perte de repères et engendrer une crise identitaire forte ${ }^{31}$.

Tous les enfants de Küper sont aujourd'hui décédés, mais son nom est encore bien vivant aux îles Salomon, porté par ses nombreux petitsenfants et arrière-petits-enfants restés à Santa Ana ou installés à la capitale. Son souvenir est lui aussi bien vivace même si, à Gupuna, on continue de l'évoquer à mi-voix. Interrogés sur les circonstances de sa mort, les villageois ont laconiquement indiqué qu'il passa de vie à trépas après avoir mangé une langouste. Au-delà du regrettable embarras gastrique que l'ingestion d'une langouste pas fraîche peut engendrer, pour les gens de Gupuna, la mort de Küper reste entourée d'un halo de mystère, tout comme celles de ses trois fils, morts, dit-on, relativement jeunes et pour des raisons mal connues ou que l'on ne souhaite pas connaître. Feinte ou sincère, cette indifférence suggère que seule une disparition prématurée et un peu énigmatique pouvait conclure l'existence d'hommes dont les actes et les choix restent eux aussi, par bien des aspects, mystérieux, obscurs, contradictoires, irrationnels ou critiquables aux yeux des Owa.

\section{BIBLIOGRAPHIE}

\section{Articles et ouvrages}

BennetT, Judith Ann, 1987. Wealth of the Solomons. A history of a Pacific archipelago, 1888-1978, Pacific Islands Monograph Series n³, Honolulu, University of Hawaii Press.

Bernatzik, Hugo, 1935a. Sudsee, Travels in the South Seas, London, Western Pacific Archives Constables \& co ltd.

_, 1935b. A Magic Isle, Owa Raha in the Solomon Archipelago, The Geographical Magazine, pp. 50-66.

-, 1935c, South Seas, New York, Henry Holt and Company.

—, 1936, Owa Raha, Wien, Bernina Verlag.
—, 1952. Canaques et Papous (Sudsee), Paris, Société Nouvelle des Éditions Self.

Burt, Ben, 1994. Tradition and Christianity, The colonial transformation of a Solomon Islands Society, Switzerland, Harwood Academic Publishers.

Coppet De, Daniel et Hugo ZEMP, 1978. 'Aré 'aré, Un peuple mélanésien et sa musique, Paris, Seuil.

Corris, Peter et Roger KeEsING, 1980. Lightning Meets the West Wind: the Malaita Massacre, Melbourne, Oxford University Press.

DAVENPORT, William, 1972. Preliminary Excavations on Santa Ana Island, Eastern Solomon Islands, Archeology and Physical Anthropology in Oceania 7 (3), pp. 165-183.

—, 1980. Male initiations in Aoriki, Expeditions 23 (2), pp. 4-19.

Fox, Charles Elliot, 1967. The story of the Solomons, Taroaniara, British Solomon Islands D.O.M. Publications.

Gapiara, Nelson, 1999. Brief history of Santa Ana [brochure non foliotée distribuée lors de la cérémonie de réouverture de l'église SSEC à Santa Ana en 1999].

Gash, Noel et Witthaker, June, 1975. A pictorial history of New Guinea, Hong Kong, The Jacaranda Press.

GoldEN, Graeme A., 1993. The early European settlers of the Solomon Islands, Melbourne, G. Golden.

Hogbin, Herbert Ian, 1939. Experiments in Civilisation. The effects of european culture on a native community of the Solomon Islands, London, Routledge \& Sons Ltd.

KeEsing, Roger, 1978-1979. Politico-Religious Movments and Anticolonialism on Malaita: Maasina Rule in Historical Perspective, Oceania 48 (1978), pp. 241-61; Oceania 49 (1979), pp. 46-73.

_, 1985. Killers, Big Men, and Priests on Malaita : Reflection on a Melanesian Troika System, Ethno$\log y 24$, pp. 237-252.

KÜPER, Geoffrey, 1937. An initiation Ceremony in the British Solomon Islands, The Native Medical Practitioner 2(4), pp. 387-98.

KÜPER, Heinrich, 1926. Tapitapi or the tattoing of Females on Santa Ana, Santa Catalina, Journal of Polynesian studies 10, pp. 1-5.

MEAD, Sydney Moko, 1973a. Material culture and art in the Star Harbour Region, Eastern Solomon Islands, Ethnography Monograph 1, Toronto, Royal Ontario Museum, pp. 7-71.

- 1973b. The last initiation ceremony in Gupuna, Santa Ana, Eastern Solomon Islands, Records of the Auckland Institute and Museum 10, pp. 69-95.

d'ailleurs les parents des îles alentours préfèrent ne pas envoyer leurs enfants. Des informateurs ont également rapporté qu'en 1986, une autre jeune fille originaire de Makira, venue passer des vacances chez des parents de Gupuna, fut elle aussi violée par plusieurs garçons de l'île.

31. Les mécanismes, enjeux et stratégies du vaste filtrage culturel dont Küper fut un agent historique mériteraient évidemment une analyse spécifique, que j'ai longuement abordée ailleurs (Revolon s.d., De l'objet sacré au curios. La production artistique comme support de la tradition dans l'est des îles Salomon (Océanie), thèse en cours de rédaction, Marseille, EHEss). 
-, 1973c. Folklore and placenames in Santa Ana, Solomon Islands, Oceania 43 (3), pp. 315-37.

VAN DEN Broek D’Obrenan, Charles, 1939. Le voyage de la Korrigane, Paris, Payot.

White, Geoffrey Miles, 1991. Identity through history, Living stories in a Solomon Islands Society, Cambridge, New York, Cambridge University Press.

Archives consultées aux Archives Nationales des îles Salomon, Honiara

British Solomon Islands Protectorate File 9/II, General Correspondance 1956-62, Santa Ana Land Disputes 1957-58: Letter from the District Com- missioner S.R. Pearson to Juridical Commissioner, October 1957.

British Solomon Islands Protectorate File 9/II, General Correspondance 1956-62, Santa Ana Land Disputes 1957-58: Letter Tedder to Allen, Special Lands Commissioner, Honiara, March 1958.

British Solomon Islands Protectorate File 9/III, Districts Reports 1919-1965, Eastern District Monthly and Quaterly reports : Monthly Report August 1920.

British Solomon Islands Protectorate File 9/III, Eastern District Annual Reports 1919-1956 : Annual Reports 1919, 1930, 1932, 1940. 